\title{
Eating with an Artificial Commensal Companion
}

\author{
Conor Patrick Gallagher \\ University College Cork \\ Cork, Ireland \\ cpg.gallagher@gmail.com
}

\author{
Radoslaw Niewiadomski \\ University of Trento \\ Istituto Italiano di Tecnologia \\ radoslaw.niewiadomski@iit.it
}

\author{
Merijn Bruijnes \\ Delft University of Technology \\ Delft, The Netherlands \\ m.bruijnes@tudelft.nl
}

\author{
Gijs Huisman \\ Amsterdam University \\ of Applied Sciences \\ Amsterdam, The Netherlands \\ g.huisman@hva.nl
}

\author{
Maurizio Mancini \\ School of Computer Science \& IT \\ University College Cork \\ Cork, Ireland \\ m.mancini@cs.ucc.ie
}

\begin{abstract}
Commensality is defined as "a social group that eats together", and eating in a commensality setting has a number of positive effects on humans. The purpose of this paper is to investigate the effects of technology on commensality by presenting an experiment in which a toy robot showing non-verbal social behaviours tries to influence a participants' food choice and food taste perception. We managed to conduct both a qualitative and quantitative study with 10 participants. Results show the favourable impression of the robot on participants. It also emerged that the robot may be able to influence the food choices using its non-verbal behaviors only. However, these results are not statistically significant, perhaps due to the small sample size. In the future, we plan to collect more data using the same experimental protocol, and to verify these preliminary results.
\end{abstract}

\section{CCS CONCEPTS}

- Human-centered computing $\rightarrow$ Human computer interaction (HCI); Interaction paradigms.

\section{KEYWORDS}

computational commensality, social robot, artificial companion, food choice

\section{ACM Reference Format:}

Conor Patrick Gallagher, Radoslaw Niewiadomski, Merijn Bruijnes, Gijs Huisman, and Maurizio Mancini. 2020. Eating with an Artificial Commensal Companion. In Companion Publication of the 2020 International Conference on Multimodal Interaction (ICMI '20 Companion), October 25-29, 2020, Virtual event, Netherlands. ACM, New York, NY, USA, 5 pages. https://doi.org/10. $1145 / 3395035.3425648$

Permission to make digital or hard copies of all or part of this work for personal or classroom use is granted without fee provided that copies are not made or distributed for profit or commercial advantage and that copies bear this notice and the full citation on the first page. Copyrights for components of this work owned by others than ACM must be honored. Abstracting with credit is permitted. To copy otherwise, or republish, to post on servers or to redistribute to lists, requires prior specific permission and/or a fee. Request permissions from permissions@acm.org.

ICMI '20 Companion, October 25-29, 2020, Virtual event, Netherlands

(c) 2020 Association for Computing Machinery.

ACM ISBN 978-1-4503-8002-7/20/10 . \$ $\$ 15.00$

https://doi.org/10.1145/3395035.3425648

\section{INTRODUCTION}

Eating together in a social setting, also referred to with the word "commensality", has a positive impact on a variety of health and wellbeing related measures, as well as food enjoyment and choice [18]. Current trends, especially in, but not limited to, Western cultures, show that people are eating, consciously or due to force majeure, an increasing number of meals alone [1, 2]. An example of the former is when people choose to eat in front of the television, or while using their smartphone; an example of the latter is elderly people living alone, or those living with physical (e.g., living away from family or being in medical self-isolation) and social (e.g., a lack of friends) barriers preventing them from experiencing commensality. According to a recent study carried out by the Oxford Economics and the National Centre for Social Research, eating meals alone is an important cause of unhappiness in UK (measured with by Sainsbury's Living Well Index in 2018 [3]). While potential negative effects of solo-eating are largely unknown, concerns have risen regarding loneliness [20], distracted eating [7] (e.g., eating in front of the TV or with a smartphone) and unhealthy food choices [18].

Recent works are starting to explore digital and computational forms of commensality [13,17], in which technology aims to replicate/emulate/enable remote forms of commensality. While eating together with others, in particular family members and friends, is something that is difficult to replace and easily missed when situations prevent co-dining (e.g., during social distancing measures and lock-downs), technological solutions could potentially help reduce negative consequences of eating alone. One particularly promising technology in this regard is social robots. In general, social robots emulate human social behaviors in order to communicate with humans, see for example the vast literature on Social Signal Processing [5]. Such robots may take diverse forms (from humanoid to abstract) and can use a variety of communication modalities (e.g., voice, touch, or movement). Social robots have been applied in a wide variety of settings and have shown to have positive effects on perceived social presence [14]. However, existing work on social robots at the dining table is relatively limited [13]. It is currently not clear how social robots could fit in during social eating settings; in other words: how do we teach social robots table manners?

In this paper, we present the first results of a study in which a simple social robot uses non-verbal behaviors to attempt to influence a person's food choice and perception. This task was proposed to make the human-robot interaction in the commensality setting 
more goal-oriented and to differentiate it from pure entertainmentoriented interaction (see, e.g., [9]). Not only do we aim to measure the user's experience with this technology, but also the ability of the robot to impact the human behaviors in a commensality scenario. Through gaining insights into how people respond to such a robot showing reactions to food choice, we hope to be able to develop artificial social agents whose role at the dining table is more fitting with that of a human co-diner.

\section{BACKGROUND}

There have been a number of attempts at making artificial companions that are present during a meal, with varying degrees of success [13]. However, Artificial Commensal Companions (ACC), that is, companions participating in eating-related activities [11], are not widely studied. There exist a few examples, but none of them is exactly fitting our definition of ACC: e.g., they can establish a conversation while the user is eating, but they are not aware of the eating itself and do not actively participate in the eating activity. In 2019, a paper proposed a speculative design prototype called FoBo, that acts and behaves like a human co-diner [9]. The robot's movements are controlled by Arduino, while sound synthesizers generate eating-specific sounds. The goal of FoBo is to provide some sort of company to the diner by participating in a social activity with the user. FoBo "eats" by picking up batteries, putting them in its belly, burping, making noises and commenting on the quality and freshness of the batteries. With FoBo, a strong emphasis is on the entertainment, to provide companionship and some aspects of commensality, making dining more fun. FoBo is not intended to influence the behavior of the user, unlike our system in its Active condition (see Section 3.1), but thanks to its ability to entertain the user, it may also be suited to such a task. By investigating interpersonal communication over a meal, Inoue [8] found out that a person who is not eating is more likely to speak, while the other person is more likely to listen, if only one side is eating a meal. This finding is the basis for the creation of an anthropomorphic agent which acts as an active listener, while also being a virtual eating companion [10]. Takahashi et al. [19] developed a virtual co-eating system with two design goals: creating "a preferable eating partner" that can also achieve an "enjoyable conversation". For the first aim, researchers exploited a $3 \mathrm{D}$ virtual character that mirrors the user, i.e., the character performs the same actions as the user to increase the character's believability. To achieve an "enjoyable conversation", the virtual partner answers to the user, talking about common topics, topics related to the meal, or just daily conversation topics.

\section{EXPERIMENT}

We designed an experiment to answer two main research questions: RQ1) is the presence of an interactive and social ACC preferred over eating alone? RQ2) can an ACC influence food choice through only its non-verbal behaviors?

\subsection{Conditions}

Participants are asked to take part in two different chocolate testing scenarios: a Baseline condition and an Active condition. The two scenarios are previously introduced to participants with written a "cover story": a food company is launching a new type of chocolate in the coming months; you are kindly asked to try and rate the taste of four types of chocolates made with slightly different ingredients; you will compare two of these chocolates types at a time, by picking chocolates from two bowls placed in front of you; you have about three minutes to freely pick from any of the bowl any number of chocolates you like. In the story, of course, we do not make any reference to the presence of the robot in the Active condition.

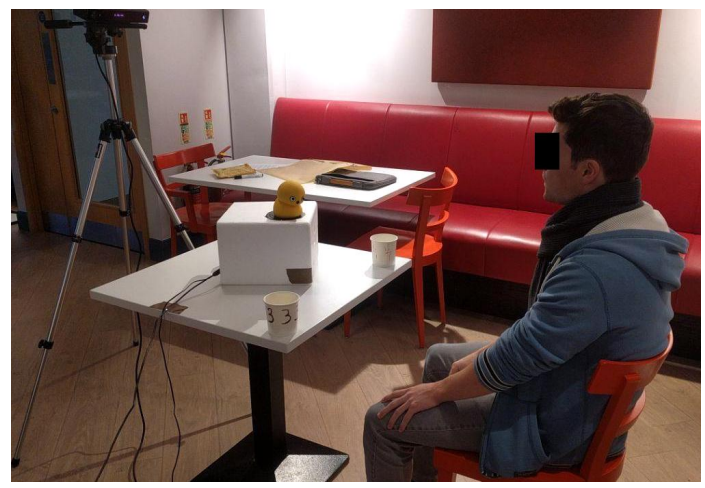

Figure 1: Experimental setup of the Active condition: the participant is seated at a dining table with two bowls with identical chocolates; the robot is placed on the table at an equal distance from the bowls, performing non-verbal behaviors to influence participant's food choice.

In the Baseline condition, the robot is not present, so the subject is seated alone at a dining table with two bowls in front of them, each containing ten identical chocolates.

In the Active condition, the subject is seated at an identical table with two new bowls in front of them, each containing ten chocolates, but this time the robot is placed on the table in front of them, as illustrated in Figure 1. The robot will try to influence the subject to eat more from a target bowl (the bowl placed on the left of the participant) and less from a non-target bowl (the bowl placed on the right of the participant).

It has been shown that it is possible to make robots capable of interactions that are "accepted" by humans while eating, i.e., that will not be perceived as inappropriate, unusual, or disturbing during food consumption [9]. While it might be relatively easy to create a humanoid robot, or a virtual agent, carrying on a conversation with humans about food quality, or providing dietary suggestions (e.g., $[12,16])$, it is still unclear what kind of non-verbal behavior ACCs should display during meals [17]. Therefore, in the Active condition, the robot is actively trying to influence the behavior of the participant through non-verbal behaviors such as gaze, head and body movements, as well as sounds (i.e., non-verbal vocalizations). The gaze behavior of the ACC was carefully designed in order to allow it to seamlessly integrate in eating-related interactions. The robot will "gaze" at the dominant hand of the user (specified prior to the beginning of the experiment scenario) for eight seconds, then it will gaze at the target bowl for three seconds, and subsequently return to gazing at the dominant hand for eight seconds, and so on. This continues for the duration of the experiment scenario, which is three minutes long. If the user takes a chocolate from the 
target bowl, the robot will randomly perform one of two reactions: a "jump" reaction, coupled with a "happy" sound, or a "head nod" reaction, coupled with a different "happy" sound, to signal that this bowl is the right choice. However, if the user takes a chocolate from the non-target bowl, the robot will make an "unhappy" sound and do a long "forward bow" sad reaction, to signal that this bowl is the wrong choice. Details about the robot system implementation can be found in [11].

\subsection{Data collection}

We collected participants' data in terms of quantitative measures and qualitative responses, as it follows:

- Chocolate Evaluation (quantitative measure): after each condition (both Baseline and Active) we counted the number of remaining chocolates in each bowl;

- Robot Evaluation (qualitative response): after experiment, we administered a survey with open-ended questions to determine the participants' social perception of the robot, as well as their opinions on the chocolate, and interaction. The open-ended questions were:

(1) What did you think of the overall experience/setup?

(2) What did you think about the chocolates?

(3) Overall, which chocolate was your favorite?

(4) What was your impression of the robot? (Like/Dislike)

(5) Did you prefer having a robot companion over no companion at all?

(6) Did you think the robot had a specific purpose, if so, what did you think it was?

(7) Would you ever consider a robot as a dinner table companion? Why/Why not?

(8) What else should the robot be able to do to be more useful for you?

(9) (Optional) Who could benefit from such a robot companion?

(10) (Optional) Do you have any other comments to share with us?

\subsection{Results}

Ten students ( 8 males, 2 females) participated in the pilot study. All of them performed both conditions. Due to external factors (i.e., the Covid-19 outbreak) we could not complete the study which was originally planned to involve 40 participants testing the two conditions in a different order. Figure 2 reports quantitative results, i.e., the number of chocolates eaten out of each of the four bowls by the participants (two bowls in the Baseline and two in the Active condition).

While there is no statistically significant difference between the bowls and conditions (possibly due to small number of participants), Figure 2 shows that in Baseline Condition the participants ate nearly the same quantity of chocolates from each bowl. In the Active condition, however, they ate fewer chocolates from the non-target bowl (i.e., the one with negative feedback) and more from the target bowl (i.e., the bowl on which the ACC was directing the attention of the user by providing positive feedback). In total, fewer chocolates were eaten in the Active condition, which might be due to the fact the participants were less hungry in that condition, or that the

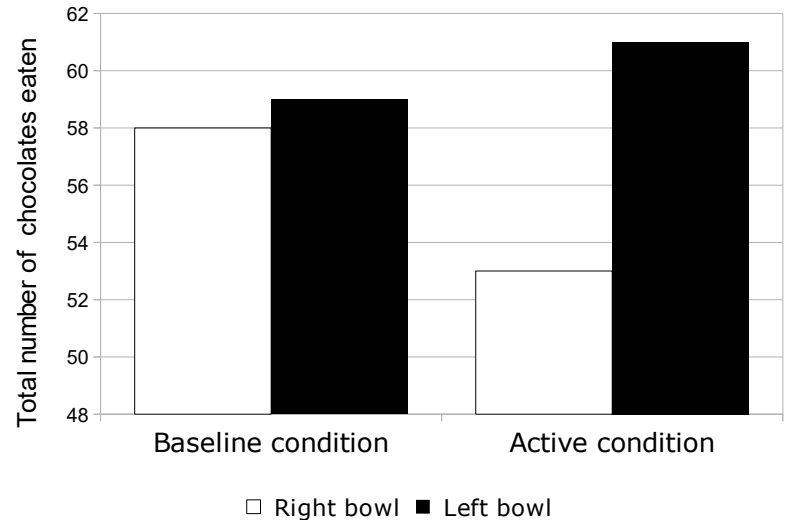

Figure 2: The total number of chocolates eaten from each bowl in Baseline and Active condition. Note, that the left bowl was the target bowl in the Active condition.

robot was engaging them and distracting them from eating (see also the participants' comments below). In the future, data from more participants need to be collected using a counter-balanced design (i.e., exchanging the order of conditions).

The surveys from six participants were used for the qualitative analysis. Figure 3 reports the word cloud of the words used by participants to answer to the open-ended questions of the survey (larger words means the word occurred with a higher frequency). It can be seen the words such as "enjoyable", "entertaining", and "fun" are larger than neutral or negative words, showing the overall positive receipt of the robot and the interaction.

In more detail, when responding to the question (4), all participants indicated to like the robot. One participant commented on the nature of the robot's behavior:

"I thought it was fun playing with gestures and seeing the robots reactions, both physically and verbally. The robot also seemed really curious about my actions"

Other participants mentioned that they appreciated the robot's presence while they were tasting the chocolates, remarking that it was enjoyable and made them feel less alone:

"I liked the robot, made it comfortable to eat as I didn't feel I was all by myself"

"I liked the robot. He made eating the chocolate more enjoyable."

In addition, when asked whether participants preferred tasting the chocolates by themselves or with the robot companion all participants indicated preferring the tasting with the robot companion present. One participant remarked:

"I preferred the robot companion, it made it less awkward than being alone and eating in public."

Regarding the question (7), majority of participants (four out of six) responded that they would consider it with one participant remarking: 
"Yeah, I think it would be a fun experience if the robot was part of a group, but if it was a one-on-one situation I'm not sure if I would enjoy it"

In contrast, the participants who responded positively indicated not having to eat alone as the primary reason for considering the robot along with its potential entertainment value. Adding speech to the robot's interactive capabilities was the main suggestion made by participants regarding additional features, which could be interpreted as participants looking for a more sophisticated interaction to reduce the feeling of eating alone. Indeed, the robot's potential to reduce feelings of loneliness was underlined when participants were asked about who could benefit from the robot's presence. Half of the participants suggested people living alone (the elderly were given as an example) could benefit from the robot.

We also asked participants what they thought the robot's purpose was. Interestingly, only one participant out of six commented that the robot attempted to "force you to pick a certain cup of chocolate". Half of participants indicated they thought the robot was their purely for entertainment, while one participant thought the robot tried to encourage more mindful eating:

"[...]with someone staring at me it caused me to eat fewer chocolates because I felt like I was being judged or something"

Finally, one participant thought that the robot was there to make participants feel more at ease and less alone while the tasted the chocolates.

Regarding the chocolates perception, all participants indicated they like the flavor and texture but none of the participants reported clear differences. However, when indicating their preference for the chocolates (i.e., "Overall, which chocolate was your favorite?" with the choice being between each of the four bowls presented during the two trials), four out of six participants indicated to prefer the chocolates in the target bowl indicated by the robot.

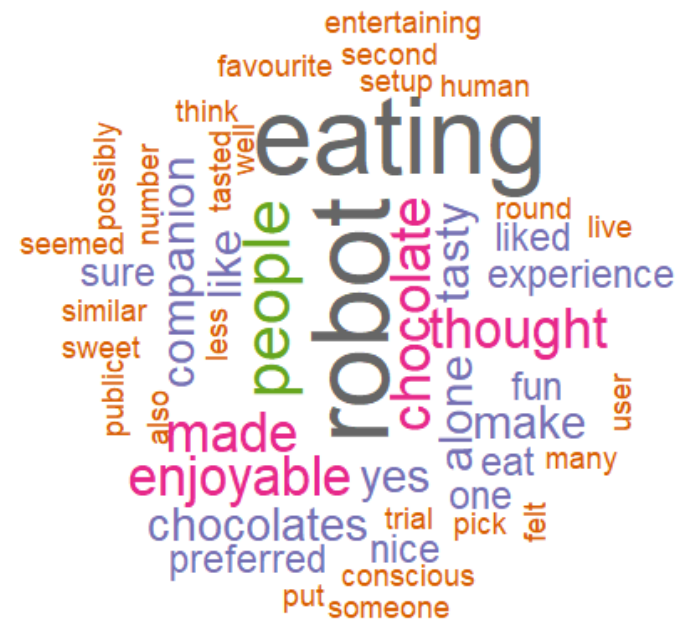

Figure 3: Word cloud of the open-ended questions.

\section{DISCUSSION}

Results from our qualitative survey indicate that the presence of an interactive and social robot is preferred over eating alone (RQ1). We speculate that the subjects would prefer to have a robot displaying active social behaviours, owing to the majority of individuals expressing a desire for the robot to be more emotive, expressive and interactive in their answers to the qualitative survey. According to the participants, the presence of the robot resulted in a more enjoyable eating experience, indicating that, perhaps, they experienced commensality. We identified that the initial social perception of the robot was favourable, with the majority of participants expressing the desire to further interact with it. They also expressed the desire to have a more interactive, and expressive robot in the future. The subjects identified the groups of people who would benefit the most from the development of such ACC: the elderly and people who live alone, which are two of the groups we expect to be most at risk of social isolation, lacking the experience of commensality.

We also speculate that the robot did manage to successfully influence the food choices and preferences of the participants (RQ2). While previous research showed that artificial agents can, in general, be persuasive $[6,15]$, in the particular context of changing the eating habits using robots, they focused mainly on verbal communication (e.g., [4]), while we focus on the non-verbal one.

\section{CONCLUSION AND FUTURE WORK}

We managed to develop a functioning ACC, capable of simulating gaze and producing emotional feedback responses based on the actions of the user. While we were not able to complete the study due to extraneous circumstances, we were able to analyse the results of our qualitative survey, and drew a number of interesting conclusions, which provided positive indications to positively answer, by running further experiments. So, we consider the study to be, at least partially, successful. The positive reaction of the participants to the Active condition (the one with the ACC demonstrating interactive and social behaviours) indicates that most individuals are comfortable with the idea of eating with an ACC in place of or in addition to eating with other individuals in a commensality setting. Going forward, there are a number of research questions that we would like to investigate in the near future:

- the participants reaction to a robot not showing any social behaviour but displaying random behaviours;

- the effect of extending the palette of robot's behaviours, e.g., by implementing speech functionality;

- whether a toy robot is the most appropriate design for an eating companion, or a more humanoid robot would prove to be more effective;

- if an ACC "consuming" food (by taking some virtual or real food and simulating eating motions) would have a greater effect on the experience of commensality, or it would be perceived as a gimmick;

- whether an ACC would encourage healthier eating habits, as we showed, at least partially, that it can influence participant's choice and perception of food. 


\section{REFERENCES}

[1] 2018. Number of single-person households in the U.S. from 1960 to 2017 (in millions). Statista - The Statistics Portal. https://www.statista.com/statistics/ 242022/number-of-single-person-households-in-the-us/. Accessed September 26, 2019.

[2] 2018. U.S. Census Bureau Releases 2018 Families and Living Arrangements Tables. https://www.census.gov/newsroom/press-releases/2018/families.html. Accessed September 26, 2019.

[3] 2020. The Sainsbury's Living Well Index. https://www.about.sainsburys.co.uk/ $\sim /$ media/Files/S/Sainsburys/living-well-index/sainsburys-living-well-indexmay-2018.pdf. Accessed February 12, 2020

[4] I. Baroni, M. Nalin, M. Coti Zelati, E. Oleari, and A. Sanna. 2014. Designing motivational robot: How robots might motivate children to eat fruits and vegetables. In The 23rd IEEE International Symposium on Robot and Human Interactive Communication. 796-801. https://doi.org/10.1109/ROMAN.2014.6926350

[5] Judee K Burgoon, Nadia Magnenat-Thalmann, Maja Pantic, and Alessandro Vinciarelli. 2017. Social signal processing. Cambridge University Press.

[6] V. Chidambaram, Y. Chiang, and B. Mutlu. 2012. Designing persuasive robots: How robots might persuade people using vocal and nonverbal cues. In 20127 th ACM/IEEE International Conference on Human-Robot Interaction (HRI). 293-300.

[7] H. S. Ferdous, B. Ploderer, H. Davis, F. Vetere, and K. O'hara. 2016. Commensality and the social use of technology during family mealtime. ACM Transactions on Computer-Human Interaction (TOCHI) 23, 6 (2016), 1-26.

[8] Tomoo Inoue 2014. Naturalistic Control of Conversation by Meal: Induction of Attentive Listening Attitude through Uneven Meal Distribution in Co-Dining. In Proceedings of the 2014 ACM International foint Conference on Pervasive and Ubiquitous Computing: Adjunct Publication (Seattle, Washington) (UbiComp '14 Adjunct). Association for Computing Machinery, New York, NY, USA, 601-606. https://doi.org/10.1145/2638728.2641341

[9] R. A. Khot, E. S. Arza, H. Kurra, and Y. Wang. 2019. FoBo: Towards Designing a Robotic Companion for Solo Dining. In Extended Abstracts of the $2019 \mathrm{CHI}$ Conference on Human Factors in Computing Systems (Glasgow, Scotland Uk) (CHI EA '19). ACM, New York, NY, USA, LBW1617:1-LBW1617:6.

[10] R. Liu and T. Inoue. 2014. Application of an Anthropomorphic Dining Agent to Idea Generation. In Proceedings of the 2014 ACM International Foint Conference on Pervasive and Ubiquitous Computing: Adjunct Publication (UbiComp '14 Adjunct).
ACM, New York, NY, USA, 607-612.

[11] Maurizio Mancini, Radoslaw Niewiadomski, Gijs Huisman, Merijn Bruijnes, and Conor Patrick Gallagher. 2020. Room for one more?-Introducing Artificial Commensal Companions. In Extended Abstracts of the 2020 CHI Conference on Human Factors in Computing Systems. 1-8.

[12] D. McColl and G. Nejat. 2013. Meal-time with a Socially Assistive Robot and Older Adults at a Long-term Care Facility. f. Hum.-Robot Interact. 2, 1 (Feb. 2013), 152-171.

[13] R. Niewiadomski, E. Ceccaldi, G. Huisman, G. Volpe, and M. Mancini. 2019. Computational Commensality: from theories to computational models for social food preparation and consumption in HCI. Frontiers in Robotics and AI 6 (2019).

[14] Andre Pereira, Rui Prada, and Ana Paiva. 2014. Improving social presence in human-agent interaction. In Proceedings of the SIGCHI Conference on Human Factors in Computing Systems. 1449-1458.

[15] Isabella Poggi, Radoslaw Niewiadomski, and Catherine Pelachaud. 2008. Facial Deception in Humans and ECAs. In Modeling Communication with Robots and Virtual Humans, Ipke Wachsmuth and Günther Knoblich (Eds.). Lecture Notes in Computer Science, Vol. 4930. Springer Berlin Heidelberg, 198-221. https: //doi.org/10.1007/978-3-540-79037-2_11

[16] Natasha Randall, Swapna Joshi, and Xiaohang Liu. 2018. Health-e-Eater: Dinnertime Companion Robot and Magic Plate for Improving Eating Habits in Children from Low-Income Families. In Companion of the 2018 ACM/IEEE International Conference on Human-Robot Interaction (Chicago, IL, USA) (HRI '18). ACM, New York, NY, USA, 361-362. https://doi.org/10.1145/3173386.3177828

[17] C. Spence, M. Mancini, and G. Huisman. 2019. Digital commensality: Eating and drinking in the company of technology. Frontiers in psychology 10 (2019), 2252.

[18] N. Stroebele and J. M. De Castro. 2004. Effect of ambience on food intake and food choice. Nutrition 20, 9 (2004), 821-838.

[19] M. Takahashi, H. Tanaka, H. Yamana, and T. Nakajima. 2017. Virtual Co-Eating: Making Solitary Eating Experience More Enjoyable. In Entertainment Computing - ICEC 2017, N. Munekata, I. Kunita, and J. Hoshino (Eds.). Springer International Publishing, Cham, 460-464.

[20] Vasoontara Yiengprugsawan, Cathy Banwell, Wakako Takeda, Jane Dixon, Samang Seubsman, and Adrian C Sleigh. 2015. Health, happiness and eating together: what can a large Thai cohort study tell us? Global journal of health science 7, 4 (2015), 270. 